\title{
USER-FRIENDLY REPORTING OF RESEARCH FINDINGS FROM A QUESTIONNAIRE
}

\author{
Andrej Šorgo \\ University of Maribor, Slovenia
}

Hooray! You have succeeded in publishing an article based on your own, yet unpublished questionnaire. This can certainly be regarded as a personal success. However, many articles based on questionnaires are not particularly reader-friendly. I am sure that these authors are not being deliberately evil; however, in their path to fame, they forget that their writing is not a secret diary but something that should communicate readily with the reader.

An enormous number of research articles in the field of education are published on a daily basis, many of them based on the application of questionnaires as research instruments. In early October 2017, a quick search of Thomson Reuters Web of Science database using the search term "education*"yielded 849,962 hits, mainly, but not exclusively listed in the following databases and categories: Science Citation Index Expanded - Education, Scientific Disciplines (40 hits) and Social Sciences Citation Index - Education \& Educational Research (235 hits), Education, Special (40 hits) Psychology, Educational (53 hits). A similar search in Elsevier's Scopus database yielded 1,900,897 hits, from 1017 journals listed in the SNIP database. Google Scholar, a search engine not protected by subscription, as is the case with academic databases, produced 5,370,000 hits for education and 4,130,000 for questionnaire. These numbers (leaving aside sources not listed in established databases, books, conference proceedings, grey-papers, duplicate titles listed in different databases and so on--just to illustrate that the number of papers is indeed huge) far exceed the range that could possibly be read by any single living person. In addition, if someone is inspired to read your particular paper, then you should think of this person as a friend, and it is not polite to be rough with friends.

Assume that you have implemented advice on how to make your research findings visible by choosing a catchy title and composing a striking initial sentence for your abstract, and have succeeded in attracting someone to open a web-page offering your abstract, along with the information that the price for your article is let's say, \$26.95. If the abstract concludes with a statement like this: "To improve $X$, we make recommendations for the future use (application, etc) of $X$ in the conclusion," you can be sure that you will make no friends this way. There are many guidelines telling us how to write a structured abstract. Briefly, the logical order of an abstract should be as follows: Background, Purpose, Sample, Methodology, Results and Conclusions.

Assume that your article using a questionnaire as a data-acquisition method has passed the abstract threshold and someone with the same problem wants to use a questionnaire to answer a similar research question. It isn't that this person doesn't trust you; however, the cornerstones of research methodology are reproducibility and replicability (Pashler \& Wagenmakers, 2012). In addition, it is impossible to verify your findings unless you include the questionnaire in the text (perhaps as an appendix or supplement) or refer directly to the full text of your instrument, if published elsewhere. At this point, I suggest using a pre-tested and applied questionnaire whenever possible. This makes it much easier to compare your findings with those achieved by the rest of the world. An extra bonus is that when you get around to writing up the discussion, this becomes a much easier task.

Most of the time, results from a questionnaire are reported in tables, which are sometimes incomprehensible. One doesn't have to seek up-to-date references to find recommendations for making tables more user-friendly. For example, Weiner's (1992) recommendations are as follows:

- Arrange the rows and columns in a table in a way that makes sense (this apply for graphs too).

Example: It is fact that statements in a questionnaire follows an order from the beginning to the end. If the authors of the questionnaire succeed in finding enough victims, they will assemble sufficient data. Nowadays, this data is usually transferred to one of a range of statistical programmes, any of which will 
ISSN 1648-3898 /Print/

ISSN 2538-7138/Online/

follow commands to produce tables from the numbers. Thanks to the admirable cut and paste function, these are easily transferred to one of the word processors and modified according rules chosen by the journal editors (e. g. APA $6^{\text {th }}$ edition). If such tables survive scrutiny by two anonymous reviewers, they will be published as part of your paper, which can be a good excuse to open a bottle of something sparkling.

However, using too many tables ends in treating the reader as an enemy. You can rest assured that almost nobody is interested in the presentation of descriptors or calculations in the order in which they were collected. Therefore, to make your tables and derived graphs user-friendly, employ sort command to arrange the calculations into an order. Size, the largest first, can be a good choice. If size is not appropriate, use some other natural order, such as time, and tell a story not a list.

- Round up your figures.

Weiner (1992) give us three reasons for this recommendation: a) Human beings cannot readily understand more than two digits; b) We can almost never justify more than two digits of accuracy statistically; c) We seldom care about accuracy to more than two decimal places.

To raise statistical literacy, I will simply quote Weiner (1992, p. 20) as a revelation:

"The standard error of any statistic is proportional to one over the square root of the sample size. God did this, and there is nothing we can do to change it. Thus, suppose we would like to report a correlation as .25. If we don't want to report something that is inaccurate, we must be sure that the second digit is reasonably likely to be 5 and not 6 or 4. To accomplish this, we need the standard error to be less than .005. But since the standard error is proportional to $1 / \sqrt{ }$, the obvious algebra $(1 / \sqrt{ } \mathrm{n} \sim .005$ à $1 / .005=200)$ yields the inexorable conclusion that a sample size of the order of $200^{2}$, or 40,000 , is required to justify the presentation of more than a two-digit correlation. A similar argument can be made for all other statistics."

As a conclusion, if readers dislike your work, it may not be because your findings are underwhelming, but simply that they have not been presented in a readily understandable manner.

\section{References}

Pashler, H., \& Wagenmakers, E. J. (2012). Editors' introduction to the special section on replicability in psychological science: A crisis of confidence? Perspectives on Psychological Science, 7 (6), 528-530.

Wainer, H. (1992). Understanding graphs and tables. Educational Researcher, 21 (1), 14-23. 